\title{
Longitudinal bulk a coustic mass sensor
}

Hales, Jan Harry; Teva, Jordi; Boisen, Anja; Davis, Zachary James

Published in:

International Solid-State Sensors, Actuators and Microsystems Conference, 2009. TRANSDUCERS 2009.

Link to article, DOI:

10.1109/SENSOR.2009.5285500

Publication date:

2009

Document Version

Publisher's PDF, also known as Version of record

Link back to DTU Orbit

Citation (APA):

Hales, J. H., Teva, J., Boisen, A., \& Davis, Z. J. (2009). Longitudinal bulk a coustic mass sensor. In International Solid-State Sensors, Actuators and Microsystems Conference, 2009. TRANSDUCERS 2009. (pp. 311-314). IEEE. https://doi.org/10.1109/SENSOR.2009.5285500

\section{General rights}

Copyright and moral rights for the publications made accessible in the public portal are retained by the authors and/or other copyright owners and it is a condition of accessing publications that users recognise and abide by the legal requirements associated with these rights.

- Users may download and print one copy of any publication from the public portal for the purpose of private study or research.

- You may not further distribute the material or use it for any profit-making activity or commercial gain

- You may freely distribute the URL identifying the publication in the public portal 


\title{
LONGITUDINAL BULK ACOUSTIC MASS SENSOR
}

\author{
J. H. Hales ${ }^{*}$, J. Teva, A. Boisen and Z. J. Davis \\ DTU Nanotech, Technical University of Denmark, Kgs. Lyngby, Denmark
}

\begin{abstract}
Design, fabrication and characterization, in terms of mass sensitivity, is presented for a polycrystalline silicon longitudinal bulk acoustic cantilever. The device is operated in air at $51 \mathrm{MHz}$, resulting in a mass sensitivity of $100 \mathrm{HZ} / \mathrm{fg}\left(1 \mathrm{fg}=10^{-15} \mathrm{~g}\right)$. The initial characterization is conducted by depositing a minute mass by means of focused ion beam. The total noise in the currently applied measurement system allows for a mass resolution of $0.4 \mathrm{fg}$ in air.
\end{abstract}

\section{KEYWORDS}

Bulk acoustic resonator, mass sensor, MEMS, silicon, capacitive readout.

\section{INTRODUCTION}

The presented research aim at developing high-Q silicon based devices for ultra sensitive mass detection in viscous fluids - initially in air and ultimately in liquids. Especially the latter is a paramount property in order to achieve the final goal of fulfilling the demand for robust real time portable diagnostic applications. We will present a mass sensor based on a longitudinal bulk acoustic cantilever (LBAC) with a Q-factor of 3100 in air. Preliminary results yield a mass sensitivity of $100 \mathrm{~Hz} / \mathrm{fg}$ and minimum detectable mass of $0.4 \mathrm{fg}$. This initial mass sensitivity characterization has been conducted by depositing a platinum compound by means of focused ion beam (FIB) equipment. The novelty of this research is comprised by the achieved high mass sensitivity and Qfactor augmented by the simple MEMS fabrication, which is CMOS compatible.

So far the highest achievable mass sensitivity, regarding mechanical resonators, has been obtained by the flexural type [1, 2]. However when turning to bio/chemical detection in higher viscous regime than vacuum, these flexural devices experience severe challenges caused by the increased hydrodynamic damping. Thus bulk acoustic technology becomes interesting especially due to the reported high Q-factors of above $10^{5}$ [3]. Previous work on resonators employing bulk acoustic mode are primarily focused on frequency reference applications aimed at the consumer electronics market $[4,5]$. Another type is the thin-film bulk acoustic resonators (FBAR), which have been utilized as mass sensors [6, 7] with point mass sensitivity in the low femtograms. The drawback of FBARs is their large footprint, compared to the presented device, thus limiting the possibility for sensor arrays. Additionally, the use of piezoelectric material such as $\mathrm{ZnO}$ and AlN increases the fabrication complexity.

\section{PRINCIPLE AND DESIGN}

The longitudinal bulk acoustic cantilever is operated, as the name indicates, in a longitudinal extensional mode, shown in figure 1(a). The two cantilevers constituting the resonator are moving along the length in anti phase motion. This mode is actuated electrostatically and the resulting resonance frequency is detected by capacitive readout between the two electrodes and the resonator as shown in figure $1(\mathrm{~b})$.

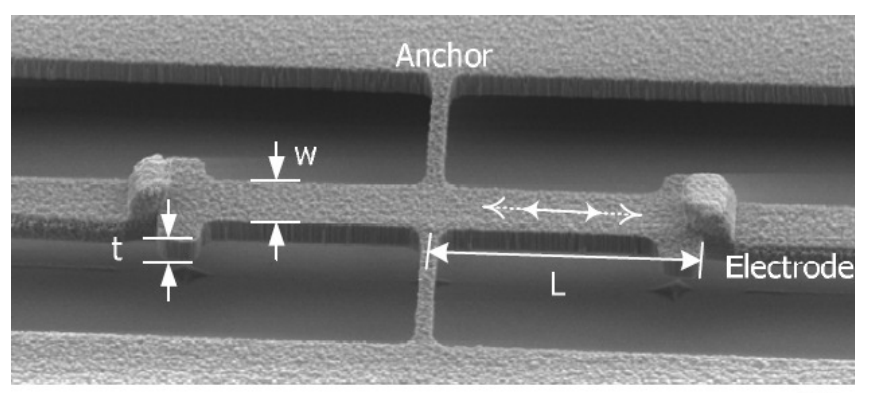

(a)

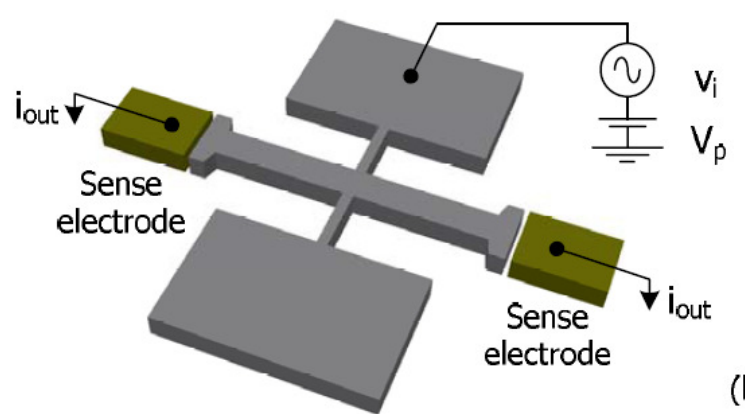

(b)

Figure 1: The scanning electron microscope (SEM) image in (a) shows the released resonator and electrodes where the mode of vibration is indicated. The dimensions are given by the length $L$ $=30 \mu \mathrm{m}$, width $w=2.5 \mu \mathrm{m}$ and the thickness $t=2.3 \mu \mathrm{m}$. The schematic in (b) shows the applied actuation and readout scheme.

By relating the strain of the beam for a given displacement to the force per area through Hooks's law and applying Newton's law of motion the resonance frequency $\left(f_{n}\right)$ for the bulk mode is obtained [10]:

$$
f_{n}=\frac{1}{4 L} \sqrt{\frac{E}{\rho}}
$$


where $E$ and $\rho$ are the Young's modulus and density respectively of the resonator material. As seen from figure 1 , an alteration (widening) of the end-part of the cantilever from the ideal beam case is implemented. This ensures good electrode-resonator overlap despite a decrease in line width during fabrication. The altered geometry results in the following analytical expression for the resonance frequency:

$$
f_{n}=\frac{1}{4 L} \sqrt{\frac{E}{\rho} \frac{w L}{2 A+w L}}
$$

where $A$ is the area of the end-part exceeding the with of the beam.

The device is configured in a 1-port measurement scheme, where the resonator is driven from the output of a network analyzer through a bias-T in order to apply the DC bias voltage $V_{P}$, as seen from figure 1(a). The output electrodes are combined and connected to the network analyzer input. The time varying motional output current $i_{\text {out }}$ measured at the output electrode is given by:

$$
i_{\text {out }}=V_{P} \frac{d C_{0}}{d l} \frac{d l}{d t}
$$

where $V_{P}$ is the applied DC voltage, $C_{0}$ is the static capacitance between the resonator and the electrodes and $d l$ is the longitudinal displacement.

The mass sensing principle of the investigated LBAC is based on change in resonance frequency $(\Delta f)$ due to mass loading given by Eq. (4) for an added mass $\Delta m$ [1].

$$
\Delta m=\frac{2 m_{e f f}}{f_{n}} \Delta f
$$

Where the effective mass $m_{\text {eff }}$ depends on the vibrational mode and $f_{n}$ is the resonance frequency of the non loaded resonator given in Eq. (2). The linear relation in (4) only holds for $\Delta m<<m_{e f f}$.

From the geometry of the cantilever end-part the effective mass $m_{\text {eff }}$ becomes:

$$
m_{\text {eff }}=\rho\left(\frac{w L}{2}+A\right) t
$$

\section{FABRICATION}

A three mask fabrication scheme comprised of five major steps is employed to realize the device. The fabrication sequence is outlined in figure 2 . The resonator and electrodes are defined by standard UV lithography using boron doped polycrystalline silicon as structural material. Initially a $5 \mu \mathrm{m}$ oxide layer followed by a $2.3 \mu \mathrm{m}$ polycrystalline silicon layer is deposited by plasma enhanced chemical vapor deposition (PECVD) and low pressure chemical vapor deposition (LPCVD), respectively. The polycrystalline silicon is subsequently heavily boron doped through solid state diffusion.

A of $0.7 \mu \mathrm{m}$ thick layer of PECVD oxide constitutes the top hard mask. Through the next step, the resonator layer is defined by reactive ion etching (RIE) of both the top oxide and the underlying polycrystalline silicon layer.

Following the deposition of $125 \mathrm{~nm}$ gap oxide (LPCVD), the second boron doped polycrystalline silicon layer comprising the electrodes are deposited and again structured by RIE.

The metal $(\mathrm{Cr} / \mathrm{Au})$ contacts are structured by standard lift-off followed by wafer dicing and resonator release in $5 \%$ buffered HF. The resulting device is shown in the SEM image in figure 1(a).
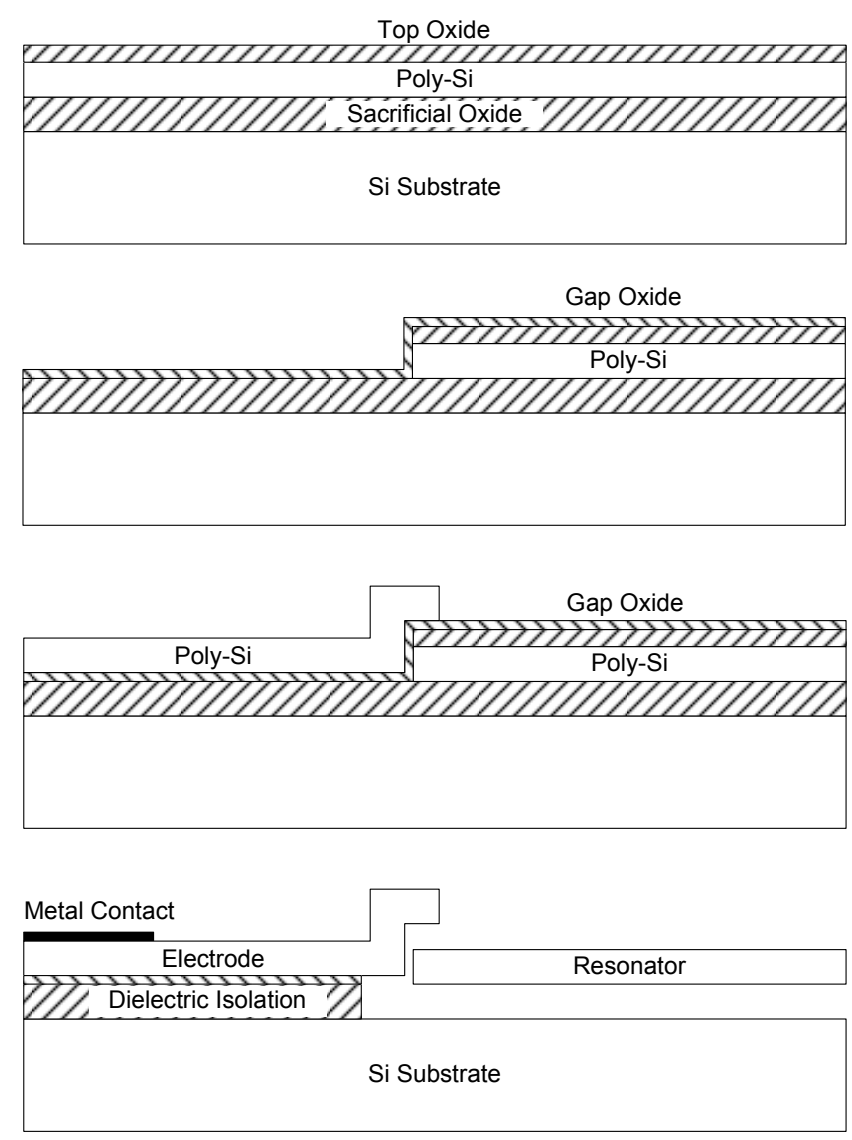

Figure 2: Cross-sectional views outlining the fabrication process. Initially the polycrystalline Si and hard mask top oxide is deposited through LPCVD processes on top of the $5 \mu \mathrm{m}$ thick PECVD oxide. Next the resonator structure is defined followed by the deposition of first the gap oxide and subsequently the second layer of polycrystalline Si. The latter is patterned to form the electrodes. Finally the metal contacts are created and the resonator is released in $\mathrm{HF}$.

The underlying $5 \mu \mathrm{m}$ thick PECVD oxide serves both to minimize the contact capacitance to the substrate and as a sacrificial layer. The thick oxide increases the yield of the subsequent device release in $\mathrm{HF}$ by minimizing the possibility of stiction between the substrate and the suspended device. 


\section{RESULTS}

From the resonance response a Q-factor of 3100, measured in air, is found for a resonance frequency of 51 $\mathrm{MHz}$. The Q-factor is on the level of what can be expected for this type of polycrystalline $\mathrm{Si}$ resonators operating in air, where the $Q x f$ value on the order of $10^{11}$ is comparable to the best mechanical mass sensing devices $[2,8,9]$. The obtained Q-factor is considered good for the specific device, but indicates that there is still room for improvement compared to similar (monocrystalline $\mathrm{Si}$ ) devices [3].

To evaluate the mass sensitivity the device is first measured in air, then a small mass of a platinum compound is deposited on the resonator structure as seen in figure 3. This is achieved through ion beam assisted deposition from an organometallic precursor being $\mathrm{C}_{5} \mathrm{H}_{5} \mathrm{Pt}\left(\mathrm{CH}_{3}\right)_{3}$ for the case of $\mathrm{Pt}$ deposition. The resulting compound consist of C, O, Pt, Ga (45-55\%, 5\%, 40-50\%, $5-7 \%$ respectively) where composition variation depends on the purity of the source and vacuum environment of the FIB system [11]. Finally, after FIB deposition the device is again measured in air.

From SEM inspection the dimension of the $\mathrm{Pt}$ deposition is obtained, resulting in an estimation of the added mass to be around $484 \pm 91 \mathrm{fg}$, where the error is based on the afore shown composition variation. Inevitably further characterization of the actual composition is needed to precisely determine the added mass $[11,12]$.
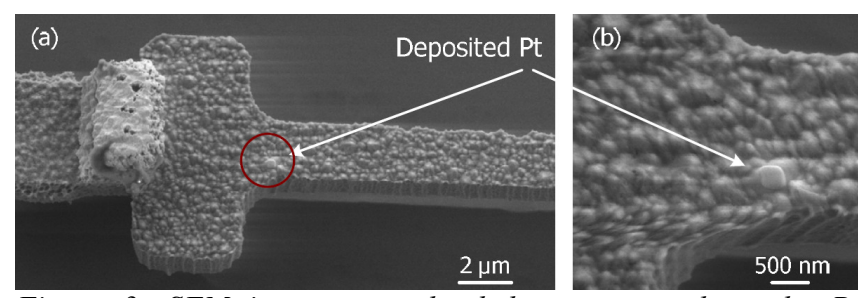

Figure 3: SEM image mass loaded resonator where the Pt compound has been deposited. The estimated mass loading is $484 \pm 91 \mathrm{fg}$.

The resonance signal before and after the mass deposition is shown in figure 4. From the expression in Eq. (4) the previous stated sensitivity of $100 \mathrm{~Hz} / \mathrm{fg}$ is obtained from an effective mass calculated based on SEM images and a density of polycrystalline silicon of $2300 \mathrm{~kg} / \mathrm{m}^{3}$ along with the resonance frequency $f_{n}=51 \mathrm{MHz}$. By looking at the phase noise of the entire system $d \Theta_{\text {noise }}$ and the slope of the phase signal at the resonance frequency $d \Theta_{\text {noise }} / d f_{n}$ the minimum detectable change in frequency for the present device and system is obtained from Eq. (6).

$$
d f_{\min }=\frac{d \Theta_{\text {noise }}}{d \Theta_{\text {noise }} / d f_{n}}
$$

This yields $d f_{\min }=45.2 \mathrm{~Hz}$ for a phase noise of 0.0094 degrees, which combined with Eq. (6) results in an experimental minimum detectable mass of $0.4 \mathrm{fg}$. This value shows that there is room for improvement since the ultimate minimum detectable mass, based purely on thermomechanical noise is $3 \mathrm{ag}\left(1 \mathrm{ag}=10^{-18} \mathrm{~g}\right)$ [1].

Employing the mass sensitivity expression in Eq. (4) and using that the decrease in frequency is $47 \mathrm{kHz}$ from the initial resonance frequency of $51 \mathrm{MHz}$, a mass change of $554 \mathrm{fg}$ is attained. This mass change is found to be in good agreement with the estimated added mass of $484 \pm 91$ fg.

The Q-factor is found to be constant before and after the deposition, indicating that the $\mathrm{Pt}$ deposition does not affect the structural parameters of the resonator, thus providing a good approach for initial mass sensitivity characterization.
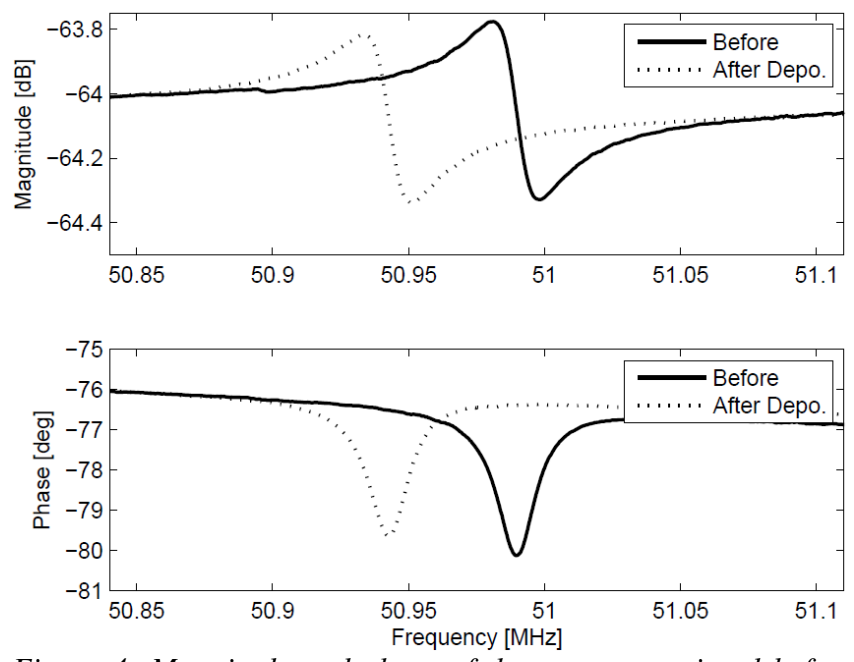

Figure 4: Magnitude and phase of the resonance signal before and after the deposition of the Pt compound-measured in air. The device is driven at an AC voltage of $650 \mathrm{mV}$ and a DC voltage of $8 \mathrm{~V}$. The change in frequency corresponds to a calculated mass change of $554 \mathrm{fg}$.

\section{CONCLUSION}

To our knowledge the obtained mass sensitivity of 100 $\mathrm{Hz} / \mathrm{fg}$ constitutes an unprecedented demonstration of point mass sensitivity in air, for bulk acoustic resonators. Through the simple characterization by means for FIB assisted Pt deposition, it has been shown that these type of resonators have great potential as mass sensors in air due to the high Q-factor and in-plane motion. Especially when the device is to be realizes in e.g. monocrystalline silicon, which will further increase the Q-factor. 


\section{REFERENCES}

[1] Ekinci, K.L., X.M.H. Huang, and M.L. Roukes, Ultrasensitive nanoelectromechanical mass detection. Applied Physics Letters, 2004. 84(22): p. 4469-4471.

[2] Ilic, B., H.G. Craighead, S. Krylov, W. Senaratne, C. Ober, and P. Neuzil, Attogram detection using nanoelectromechanical oscillators. Journal of Applied Physics, 2004. 95(7): p. 3694-3703.

[3] Mattila, T., J. Kiihamäki, T. Lamminmäki, O. Jaakkola, P. Rantakari, A. Oja, H. Seppä, H. Kattelus, and I. Tittonen, A $12 \mathrm{MHz}$ micromechanical bulk acoustic mode oscillator. Sensors and Actuators A: Physical, 2002. 101(1-2): p. 1-9.

[4] Clark, J.R., W.T. Hsu, M.A. Abdelmoneum, and C.T.C. Nguyen, High-Q UHF micromechanical radial-contour mode disk resonators. Journal of Microelectromechanical Systems, 2005. 14(6): p. 1298-1310.

[5] Khine, L., M. Palaniapan, and W.K. Wong. 6Mhz Bulk-Mode Resonator with $Q$ Values Exceeding One Million. 2007.

[6] Campanella, H., A. Uranga, A. Romano-Rodriguez, J. Montserrat, G. Abadal, N. Barniol, and J. Esteve, Localized-mass detection based on thin-film bulk acoustic wave resonators (FBAR). area and mass location aspects. Sensors \&amp; Actuators: A. Physical, 2008. 142(1): p. 322-328.
[7] Zhang, H., M.S. Marma, E.S. Kim, C.E. McKenna, and M.E. Thompson, A film bulk acoustic resonator in liquid environments. Journal of Micromechanics and Microengineering, 2005. 15(10): p. 1911-1916.

[8] Yang, Y.T., C. Callegari, X.L. Feng, K.L. Ekinci, and M.L. Roukes, Zeptogram-scale nanomechanical mass sensing. Nano Letters, 2006. 6(4): p. 4 pp.

[9] Burg, T.P., M. Godin, S.M. Knudsen, W. Shen, G. Carlson, J.S. Foster, K. Babcock, and S.R. Manalis, Weighing of biomolecules, single cells and single nanoparticles in fluid. Nature, 2007. 446(7139): p. 1066-1069.

[10] Weaver, W., JR, S. P. Timoshenko, and D. H. Young. Vibration problems in engineering. John Wiley \& Sons, 1990.

[11] Langford, R.M., T.X. Wang, and D. Ozkaya, Reducing the resistivity of electron and ion beam assisted deposited Pt. Microelectronic Engineering, 2007. 84(5-8): p. 784-788.

[12] Vila, A., F. Hernandez-Ramirez, J. Rodriguez, O. Casals, A. Romano-Rodriguez, J.R. Morante, and M. Abid, Fabrication of metallic contacts to nanometre-sized materials using a focused ion beam (FIB). Materials Science \&amp; Engineering C, Biomimetic and Supramolecular Systems, 2006. 26(5-7): p. 1063-1066.

\section{CONTACT}

*J. H. Hales, tel: +45 72202756; jan.hales@ gmail.com 